\title{
A Standalone Content Sharing Application for Spontaneous Communities of Mobile Handhelds*
}

\author{
Amir Krifa, Mohamed Karim Sbai, Chadi Barakat, Thierry Turletti \\ Planète Project-team, INRIA, France \\ \{akrifa, mksbai, cbarakat, turletti\}@sophia.inria.fr
}

\begin{abstract}
This demo illustrates the benefits of BitHoc, a standalone protocol for content sharing among spontaneous communities of mobile handhelds using wireless multi-hop connections. BitHoc is a Trackerless BitTorrent-like application adapted to mobile wireless ad-hoc networks(MANET). The current BitHoc architecture is composed of three principal components: a content sharing service, a membership management service and a content discovery service. The present demo highlights the efficiency of the BitHoc package in dealing with diverse challenges encountered in the MANET environment. Our solution considers the following issues: routing overhead, sharing opportunities and mobility of nodes. In order to validate the feasibility of our application and evaluate its performance, we consider a test-bed composed of PDAs and smartphones equipped with WIFI adapters and Windows Mobile 6 operating system.
\end{abstract}

\section{Categories and Subject Descriptors}

H.4 [Information Systems Applications]: Miscellaneous; D.2.10 [Software Engineering]: Design

\section{General Terms}

Algorithms Design Experimentation

\section{INTRODUCTION}

Content sharing is currently a universal concern among computer users and has become soon an important requirement for mobile handheld users. In fact, nowadays, the market proposes a variety of mobile devices offering user-friendly interfaces, long-life battery autonomy, sufficient computational power and efficient wireless connectivity. This tremendous advancement triggers the necessity of supporting the very fashionable desktop applications in such mobile environments. Indeed, thanks to the efficient wireless connectivity offered by mobile devices (PDAs, smartphones...), users are frequently brought to locate and share content of interest(data, photos, videos, etc) with other members of the same spontaneous community. There is a strong need to organize the communication overlay among nodes in a way to

\footnotetext{
${ }^{*}$ This work has been supported by the ITEA European project on experience sharing in mobile communities (Expeshare).
}

Copyright is held by the author/owner(s). MobiHeld'09, August 17, 2009, Barcelona, Spain. ACM 978-1-60558-444-7/09/08. distribute fairly the burden of data sharing among the set of participants while aiming to decrease the global download time. P2P file sharing solutions are good candidates for such infrastructureless networks as they are based on multisourcing which balances resources consumption among peers and reduces the dependency on any central entity. But unfortunately, $\mathrm{P} 2 \mathrm{P}$ content sharing applications developed for the Internet cannot directly be plugged and used into mobile devices [4]. Indeed, on one hand, these algorithms are not adapted to the constraints of multi-hop wireless networks. For example, it is known that in a resource constrained environment, the choice of the peers to whom to connect cannot be done independently of information on the underlying dynamic topology[4]. Moreover, centralized peer management approaches like the tracker used in BitTorrent do not perform well in such environment as the tracker can be either far away or even invisible by some peers because of disconnections. Furthermore, computer users rely on Internet search engines and dedicated desktop applications to look for the content they are willing to share. This approach becomes obsolete in the case of a spontaneous MANET based community and thus, a dedicated distributed content discovery approach must be provided. On the other hand, from a technical point of view, limited Software Development Kits (SDK) proposed for mobile handhelds represent only a subset of classical SDK(s) used for desktops which leads to incompatibility problems.

This demo presents BitHoc, an open-source standalone software solution for content sharing in MANETs that overcomes the challenges aforementioned. Our architecture consists of three main components: a content discovery service (BitHoc search engine), a membership management service (distributed BitHoc tracker) and a content sharing service (BitHoc client[3]). We developed our software on mobile devices having Windows Mobile 6 as an operating system and equipped with WIFI adapters. Our test-bed allows us to experiment with the different features of our solution and to compare our version of the BitTorrent's algorithms to the ordinary Internet one when deployed in MANET.

\section{BITHOC ARCHITECTURE}

Figure 1 depicts the principal components of the BitHoc architecture and the interactions between them. We illustrate these interactions through two typical usage scenarios:

\subsection{Content Publishing and Discovery}

A user willing to share some content with the members of his community needs to indicate to the BitHoc client the location of the content in the mobile device file system. First, 


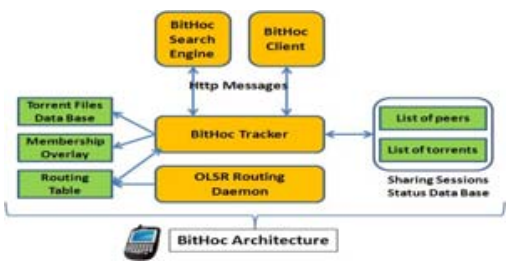

Figure 1: BitHoc Architecture

the client creates a meta-info file (torrent file) that identifies in a unique manner a sharing session of the content. After that, the user publish (locally) the new torrent file and a short text description of the related content using the BitHoc search engine service, which will update the local torrent files database maintained in the underlying BitHoc tracker via HTTP messages. A remote user, willing to share the same content, has to use the BitHoc search engine to find and download the torrent file. He specifies for that the name of the content or some key words related to its description. The request is sent via HTTP messages to its local tracker which looks for the closest match in its local database. If there are no matches, it forwards the HTTP request to the other trackers in the discovery overlay. Then it presents the received results through an ergonomic user interface (see Figure 2). Based on the details of received answers (fitness to the search, number of peers involved in the sharing session, number of seeders, and number of leechers ...), the user can choose the torrent file to download, then start the content sharing using the BitHoc client.

\subsection{Content sharing}

Before starting a new sharing session, the user can choose between two versions of BitTorrent algorithms: The classical version [2] and our version adapted to mobile ad-hoc networks [4]. Moreover, the BitHoc client offers a Wizard allowing the user to configure the parameters of BitTorrent (communication ports, choking slot duration, $\min / \max$ number of peers, etc). Once the torrent file is obtained the BitHoc client can start the sharing session where it can either play the role of a leecher or a seed. It contacts periodically the local BitHoc tracker to get the current list of members of the same content sharing session (torrent). Using this list and the routing table, it manages the connections with the interested peers. Briefly a client implementing our algorithms exchanges pieces with close peers and only seeds distribute pieces across the network. Note that we allow the user to pause or resume the download while conserving the session context. He can also monitor in real time the status of the session (downloaded bytes, uploaded bytes, numbers of leechers, number of seeders, elapsed time, etc). Figure 3 shows a screenshot of the BitHoc client. ${ }^{1}$

\section{EXPERIMENTATION AND DEMO}

\subsection{Test-bed description}

Our wireless ad-hoc network consists of 14 mobile devices including 7 PDAs (HP iPAQ 214) and 7 smartphones (HP iPAQ 614c). Each handheld is equipped with an IEEE802.11b wireless card. The characteristics of the two types of devices are detailed in[3]. The ad-hoc connectivity is maintained

\footnotetext{
${ }^{1}$ More screenshots of our application are available at[1].
}

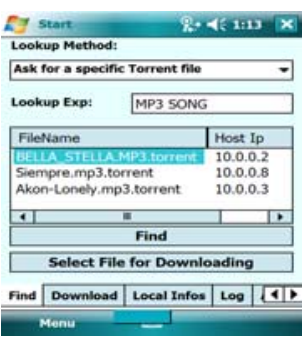

Figure 2: Search Engine

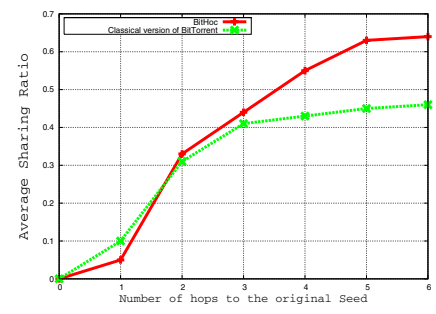

Figure 4: Sharing Ratio

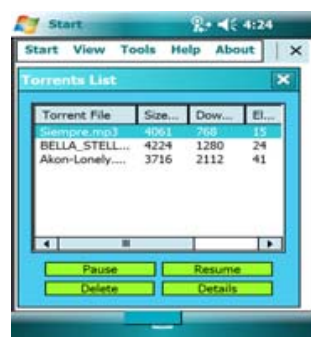

Figure 3: BitHoc Client

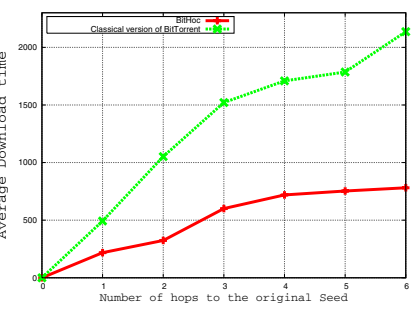

Figure 5: Download Time thanks to OLSR daemons run by the different devices. In our experiments, we constructed several network topologies containing a maximum of 6 hops. The objective of the realized swarm is to download a 4 MB MP-3 content. All PDAs were supposed to participate to the sharing of the file. The original seed of the content was chosen randomly among the set of the 14 PDAs.

\subsection{Some experimentation results}

The metrics tracked during our experiments was the download time and average sharing ratio of nodes. We define $R_{h}$ as the sharing ratio of peers located at $h$ hops from the original seed. It measures the level of reciprocity between downloads and uploads. In the ideal case, the ratio is equal to 1 . The two versions of BitTorrent have been tested and the results are presented in Figures 4 and 5. Figure 4 shows a dramatic increase of sharing opportunities when our adapted version is deployed. The routing overhead yielded by the classical version makes any gain obtained by important diversification of pieces negligible. Our method finds the good equilibrium between sharing and diversification. Figure 5 shows that BitHoc outperforms the classical version of BitTorrent in terms of download time. It is accordance to our research results[4]. More information about our research, our experiments and our GPL licensed open-source code can be found on the BitHoc web site [1].

\section{REFERENCES}

[1] BitHoc: http://planete.inria.fr/bithoc/.

[2] Bittorrent protocol: http://wiki.theory.org/bittorrentspecification.

[3] A.Krifa, M.K.Sbai, C.Barakat, and T.Turletti. BitHoc: A content sharing application for wireless ad-hoc networks, demo description in IEEE Percom, Texas.

[4] M.K.Sbai, C.Barakat, J.Choi, A. Hamra, and T.Turletti. Adapting BitTorrent to wireless ad-hoc networks, in Ad-Hoc Now, Sophia Antipolis. 